\title{
Composition as identity, now with all the pluralities you could want
}

\author{
Jonathan D. Payton ${ }^{1}[$
}

Received: 24 May 2020 / Accepted: 3 April 2021 / Published online: 7 May 2021

(c) The Author(s), under exclusive licence to Springer Nature B.V. 2021

\begin{abstract}
According to 'composition as identity' (CAI), a composite object is identical to all its parts taken together. Thus, a plurality of composite objects is identical to the plurality of those objects' parts. This has the consequence that, e.g., the bricks which compose a brick wall are identical to the atoms which compose those bricks, and hence that the plurality of bricks must include each of those atoms. This consequence of CAI is in direct conflict with the standard analysis of plural definite descriptions (and hence with the standard plural comprehension schema which uses it). According to that analysis, the denotation of 'the bricks' can include only bricks. It seems, then, that if CAI is true, 'the bricks' doesn't denote anything; more generally, if CAI is true, there are fewer pluralities than we ordinarily think. I respond to this argument by developing an alternative analysis of plural descriptions (and an alternative comprehension schema) which allows the denotation of 'the bricks' to include non-bricks. Thus, we can accept CAI, while still believing in all the pluralities we could want. As a bonus, my approach to plural descriptions and plural comprehension blocks recent arguments to the effect that CAI entails compositional nihilism.
\end{abstract}

Keywords Mereology · Composition as identity · Plural comprehension · Collapse · Mereological nihilism

\section{Introduction}

I defend the view that composition is identity (CAI): a whole is identical to its parts (in the sense of being identical to all of them taken together, not to each of them individually). Sider $(2007,2014)$ and others have raised a serious problem for CAI: while the view can only be formulated using the resources of plural first-order

Jonathan D. Payton

jonathanpayton@bilkent.edu.tr

1 Department of Philosophy, Faculty of Humanities and Letters, Bilkent University, 06800 Bilkent, Ankara, Turkey 
languages, natural ways of understanding and applying those resources seem to rule it out. In particular, plural definite descriptions-e.g. 'the bricks which form the wall'-don't function as you'd expect (and as a standard plural comprehension schema requires), if CAI is true. Thus, it seems that if CAI is true then many of the pluralities we believe in (and which are required by the comprehension schema) don't exist (Sect. 3).

In this paper, I develop an alternative approach to both plural descriptions and plural comprehension, which is compatible with CAI but motivated on independent linguistic grounds (Sects. 4-7). My approach has advantages over alternatives (Sect. 8), and also blocks recent arguments that CAI entails compositional nihilism (Sect. 9).

\section{Set-Up}

I begin with a sketch of the plural language I'll be using, and a formal statement of CAI. ${ }^{1}$

In addition to singular terms (' $a$ ', ' $b$ ', etc.) which can only denote individuals, my language contains 'inclusively' plural terms (' $a a$ ', ' $b b$ ', etc.) which can denote either individuals or pluralities thereof. (Following Calosi (2016: p. 223), say that $a a$ denotes a 'proper plurality' if it denotes many individuals, and an 'improper plurality' if it denotes only one.)

My language also contains a term-forming operator, '@'. '@’ takes two old terms to a new term which denotes together what the old ones denote individually (e.g. ' $a @ b$ ' denotes $a$ and $b)^{2}$.

With plural terms come (inclusive) plural variables (' $x x$ ', ' $y y$ ', etc.), which can take either singular or plural terms as substitutions. (' $\exists x x F x x$ ' is read as 'Some thing(s) is/are $F$ ' and is implied both by ' $F a$ ' and by ' $F a a$ '.)

A plural predication, ' $\mathrm{Faa}$ ', has two readings. On the 'distributive' reading, $\mathrm{Faa}$ ' implies ' $F a$ ' for each $a$ among $a a$ (e.g. 'Alice and Beth are people' implies both 'Alice is a person' and 'Beth is a person'). On the 'collective' reading, there's no such implication (e.g. 'Alice and Beth lifted a piano' implies neither 'Alice lifted a piano' nor 'Beth lifted a piano').

Finally, I add a two-place predicate, ' $\leqslant$ ', which denotes what I'll call 'the inclusion relation': ' $a a \leqslant b b$ ' says that $a a$ is/are included in $b b$. ' $\leqslant$ ' is read distributively at its first argument-place and collectively at its second: ' $a a \leqslant b b$ ' implies ' $a \leqslant b b$ ', for any $a$ among $a a$; but it doesn't imply ' $a a \leqslant b$ ' for any $b$ among $b b$. Inclusion is assumed to be both reflexive and transitive, and is used to regiment the phrases 'is

\footnotetext{
${ }^{1}$ See McKay (2006), Oliver and Smiley (2016), and Yi $(2005,2006)$ for more detailed discussion of plural languages.

${ }^{2}$ On one view, if ' $a @ b$ ' denotes $a$ and $b$, then it denotes each of them individually. On a competing view, there's no such implication, and indeed, if ' $a @ b$ ' denotes $a$ and $b$ then it doesn't denote either of them individually. I remain neutral on this dispute; see Oliver and Smiley (2016: ch. 6) for discussion.
} 
one of' and 'are among'. (E.g. that Alice is one of Alice and Beth is regimented as ' $a \leqslant a @ b ')^{3}$

I use inclusion to define an identity predicate, ' ='

Definition of ' $=$ '

$a a=b b \equiv_{\mathrm{df}}(a a \leqslant b b \& b b \leqslant a a)$

Identity is mutual inclusion. Since ' $\preccurlyeq$ ' is read collectively at its second argumentplace, we can use this predicate to capture the idea that wholes are identical to their parts collectively, though not individually: ' $a @ b=c$ ' doesn't imply ' $a=c \& b=c$ '.

The resources of a plural language allow us to give a standard definition of 'mereological fusion':

\section{Definition of ' $F u$ '}

$F u(a, b b) \equiv_{\mathrm{df}} \forall x(x \leqslant b b \supset x \leq a) \& \forall y(y \leq a \supset \exists z(z \leqslant b b \& y \circ z))$

$a$ is a fusion of $b b$ (or, $a$ fuses $b b$ ) just in case: (i) everything included in $b b$ is a part of $a$; and (ii) every part of $a$ overlaps something included in $b b .{ }^{4}$ Now, a mereological atom (i.e. an object whose only part is itself, and so lacks proper parts) is its own fusion, by this definition. So, I say that a composite object is a fusion with two or more proper parts, and I formulate CAI as the general claim that a fusion is (collectively) identical to the thing(s) it fuses.

\section{Composition is Identity (CAI) ${ }^{5}$}

$\forall x \forall y y(F u(x, y y) \supset x=y y)$

\section{Tensions}

While we needed plural resources to formulate CAI, Sider $(2007,2014)$ and others have shown that CAI conflicts with natural approaches to the meaning and logic of plural expressions. In particular, it conflicts with natural approaches to (i) the analysis of 'distributive' plural definite descriptions, and (ii) the formulation of a plural comprehension schema.

Informally, ' $F$ ' is read distributively just in case ' $F a a$ ' implies ' $F a$ ' for each of $a a$, and collectively otherwise. Using the inclusion predicate ' $\preccurlyeq$ ', we can give a formal treatment of this distinction:

\footnotetext{
3 Some distinguish more sharply between 'is one of', which only takes singular terms at its first argument-place, and 'are among', which only takes plural terms at its first argument-place (McKay, 2006: ch. 6; Yi, 2005: pp. 485-486). See Oliver and Smiley (2016: pp. 108-109) for discussion.

4 overlaps $b$ just in case they have a part in common.

5 CAI is often understood to imply the converse conditional: if $x=y y, x$ fuses $y y-$ see, e.g., Bohn (2014) and Wallace (2011). That conditional won't matter for our purposes.
} 


\section{Distributivity and Collectivity}

(i)

' $F$ ' is read distributively iff ' $F a a \supset \forall x x(x x \leqslant a a \supset F x x)$ ' is true

' $F$ ' is read collectively iff it isn't read distributively

With this treatment of collective and distributive predication in place, we can also give a formal analysis of plural definite descriptions, i.e. phrases of the form 'the $F$ s'.

For our purposes, I'll focus on cases where ' $F$ ' is understood to distribute over the objects described: 'the $F$ ' denotes, not the things which are collectively $F$, but the things which are, so to speak, individually $F .^{6}$ Intuitively, in such cases, 'the $F$ ' denotes those $x x$ such that every $F$ is one of them and every one of them is an $F$-e.g. 'the books on my shelf' denotes the things which are books on my shelf, and nothing else. Letting ' $\gamma x x(F x x)$ ' be a predicate corresponding to predicative uses of 'the $F \mathrm{~s}$ ' and ' $1 x x(F x x)$ ' a term corresponding to referential uses:

\section{Plural Definite Descriptions}

(i) ' $\gamma x x(F x x) a a$ ' is true iff ' $\forall y y(y y \leqslant a a \equiv F y y)$ ' is true

(ii) If there is/are some $a a$ such that $\gamma x x(F x x) a a$, then ' $1 x x(F x x)$ ' denotes $a a$. Otherwise, ' $x x(F x x)$ ' denotes nothing

$a a$ satisfy the predicate 'the books on my shelf' just in case $a a$ include(s) all and only the books on my shelf. And the term 'the books on my shelf' denotes $a a$ just in case they satisfy that predicate.

We can use this analysis of plural descriptions to formulate a comprehension schema for our plural language:

\section{Comprehension}

$\exists x \varphi x \supset \exists x x \forall y(y \leqslant x x \equiv \varphi y)$

(' $\varphi$ ' is any formula in which ' $x$ ', but not ' $x x$ ', occurs free.) The idea behind Comprehension is that, if there's at least one individual $\varphi$-er, then there's such a plurality as the individual $\varphi$-ers, or the individuals which are $\varphi .^{7}$ This is highly intuitive: if at least one individual satisfies ' $\varphi$ ', we ought to be able (in principle) to 'circumscribe' all such individuals, i.e. to single out the $\varphi$-ers as a plurality.

Comprehension is also valuable for certain theoretical purposes, e.g. in giving a first-order semantics for higher-order languages. If ' $F$ ' is satisfied by at least one individual, then by Comprehension there exists a plurality of individuals which satisfy it. We can treat this plurality, rather than the set or class of $F$-individuals, as the semantic value of ' $F$ '. We needn't treat quantification into predicate position as quantification over sets or classes.

\footnotetext{
${ }^{6}$ I say 'so to speak' because, as we'll see, we can introduce the description 'the $F$ s' in cases where ' $F$ ' applies collectively to pluralities of individuals, but not to any individual.

${ }^{7}$ Notice, it's equivalent to: $\exists x x(\exists x(x=x x) \& \varphi x x) \supset \exists y y \forall z z(z z \leqslant y y \equiv(\exists z(z=z z) \& \varphi z z))$.
} 
Unfortunately, Comprehension and the analysis of plural descriptions on which it's based are in tension with CAI.

Taking descriptions first, CAI implies that every fusion fuses at most one plurality $^{8}$ :

\section{Uniqueness}

$\forall x \forall x x(F u(x, x x) \supset \forall y y(F u(x, y y) \supset x x=y y))$

But Uniqueness makes trouble for plural descriptions. An object can be composed of different things at different 'levels of composition'. E.g., a brick wall is composed of some bricks, which are in turn composed of some atoms. Thus, the wall fuses both the atoms and the bricks. If Uniqueness is true, then the atoms are the bricks. By the definition of ' $=$ ', each plurality is included in the other, and so everything included in the atoms is included in the bricks, and vice-versa. More formally:

$$
\begin{aligned}
& \forall x(x \leqslant \iota x x(A x x) \supset x \leqslant \iota x x(B x x)) \\
& \forall x(x \leqslant \iota x x(B x x) \supset x \leqslant \iota x x(A x x))
\end{aligned}
$$

The tension with our analysis of plural descriptions is clear. On that analysis, 'the bricks' denotes a plurality that includes all and only bricks. Since no atom is a brick, no atom is included in the bricks, contra (1). Likewise, 'the atoms' denotes a plurality that includes all and only atoms. Since no brick is an atom, no brick is included in the atoms, contra (2). Therefore, the atoms can't be the bricks, contra CAI.

Generalizing: an object can be composed of $F \mathrm{~s}$ and of non- $F \mathrm{~s}$ at different levels of composition. If CAI is true, then those $F$ s are identical to those non- $F$ s, and so each $F$ is included in the non- $F \mathrm{~s}$, and each non- $F$ is included in the $F$ s. But that's impossible, given our analysis of plural descriptions.

For similar reasons, CAI is in tension with Comprehension. Comprehension has the following instance:

$$
\exists x B x \supset \exists x x \forall y(y \leqslant x x \equiv B y)
$$

If there's at least one brick, then there are such things as the bricks, i.e. some $x x$ which include all and only bricks. But if CAI is true, then (3) is false. By CAI, each brick is identical to the atoms which make it up. But then, any plurality which includes a brick must also include each of those atoms. No plurality can satisfy the condition laid down in (3).

Generalizing: an $F$ can be composed of non- $F$ s. If CAI is true, that $F$ is identical to those non- $F$ s, so any plurality which includes that $F$ must also include those

\footnotetext{
${ }^{8}$ If $x$ fuses $x x$ and $x$ fuses $y y$, then by CAI, $x=x x$ and $x=y y$; by the transitivity of identity, $x x=y y$.
} 
non- $F$ s. Thus, contra Comprehension, there can be no such things as the Fs, no plurality which includes all and only $F$ s. $^{9}$

Thus, the defender of CAI must apparently deny that ordinary plural descriptions like 'the bricks' refer (since no plurality could satisfy their reference conditions) and reject the highly intuitive (and theoretically useful) idea behind Comprehension.

These two problems are related. Our formulation of Comprehension was based on our analysis of plural descriptions, which in turn was based on our formal treatment of the collective/distributive distinction. All of these were seemingly natural. However, in Sect. 4-6 I'll argue that we should revise them all. I'll propose an alternative approach to distributive predication (Sect. 4) and use it to develop an alternative analysis of plural descriptions (Sect. 5) and an alternative comprehension schema (Sect. 6), each of which is acceptable to a defender of CAI, but also motivated on independent grounds.

\section{Distributive predication revisited}

It seems that the truth of a plural predication can depend on how we 'carve up' the relevant plurality. Consider (4):

$$
\begin{aligned}
& \text { Rogers, Hart, and Hammerstein wrote musicals } \\
& W\left(r @ h_{1} @ h_{2}\right)
\end{aligned}
$$

Rogers, Hammerstein, and Hart never wrote a musical collectively, and none of them ever wrote one individually. Thus, whether we treat 'wrote musicals' as collective or distributive, in the sense we gave these phrases in Sect. 3, (4) comes out false. But it seems true, since Rogers wrote musicals together with each of Hart and Hammerstein. ${ }^{10}$

We can account for the truth of (4) by introducing covers to the semantics of plural expressions (Gillon, 1992; Schwartzchild 1996). Covers, like worlds and times, are indices with respect to which sentences are evaluated. I take a cover to be a function, $\delta_{i}$, that maps every collection in the domain, $a a$, to some (possibly overlapping) collections in the domain, $b b_{1}, \ldots, b b_{n}$, such that $b b_{1} \ldots b b_{n}$ are collectively identical to $a a$. Thus, if $b b_{1}, \ldots, b b_{n}$ are all the values of $\delta_{i}(a a)$, then $a a=b b_{1} @ \ldots @ b b_{n}$.

\footnotetext{
9 Variants of these arguments appear in Sider (2007: pp. 57-59, pp. 63-66; 2014: pp. 215-216) and are discussed in Calosi (2018: pp. 282-287), Carrara and Lando (2017: pp. 510-511), Cotnoir (2013: pp. 313-317), and Loss (2019: p. 4). Sider's presentation uses a principle, Collapse, which I haven't discussed (although see Sect. 9), and a principle of unrestricted fusion. My presentation avoids these complications.

10 Objection: (4) is true on the distributive reading; ' $a$ wrote musicals' doesn't imply that $a$ wrote them on their own.

Reply: Other sentences more clearly illustrate the phenomenon in which I'm interested - e.g. 'Three architects designed four buildings', which can be true even if (i) there are no four buildings such that the three architects worked collectively on each of them and (ii) none of the three architects worked on four buildings, either individually or in a team. But these involve additional complexities - e.g. the interplay between the determiner phrases 'three architects' and 'four buildings' - so I'll assume, if only for simplicity, that (4) is false on the distributive reading.
} 
A cover is a multivalued function: $\delta_{\mathrm{i}}$ maps $a a$ to each of $b b_{1}, \ldots, b b_{n}$ individually, not to all of them collectively. Thus, if $\delta_{\mathrm{i}}$ maps $a a$ to $b b_{1}$ and $b b_{2}$, it doesn't follow that $b b_{1} @ b b_{2}$ are a value of $\delta_{\mathrm{i}}(a a)$; all that follows is that $b b_{1}$ is/are a value of $\delta_{\mathrm{i}}(a a)$ and that $b b_{2}$ is/are, too. ${ }^{11}$ I refer to the values of $\delta_{\mathrm{i}}(a a)$ using commas (' $\left.b b_{1}, \ldots, b b_{n}{ }^{\prime}\right)$ rather than the term-forming operator '@' (' $b b_{1} @ \ldots @ b b_{n}$ ') to indicate this.

Since covers are indices, a sentence could be true with respect to some covers and not others. E.g., let $\delta_{1}$ map the plurality of Rogers, Hart, and Hammerstein to each of the men individually, and $\delta_{2}$ map that plurality to (i) the plurality of Rogers and Hart and (ii) the plurality of Rogers and Hammerstein-i.e. $\delta_{1}\left(r @ h_{1} @ h_{2}\right)=r, h_{1}, h_{2}$ while $\delta_{2}\left(r @ h_{1} @ h_{2}\right)=r @ h_{1}, r @ h_{2}$. Then, we might say, (4) is true when evaluated with respect to $\delta_{2}$ but false when evaluated with respect to $\delta_{1}$.

So far, this is merely suggestive. Why does (4) get different truth-values when evaluated with respect to these different covers? My suggestion is that we revise our approach to collective and distributive predication.

In Sect. 3, we understood distributive predication in terms of inclusion: ' $F$ ' is read distributively just in case ' $F a{ }^{\prime}$ ' implies ' $F a$ ', for every $a$ included in $a a$. This was meant to capture the intuitive, informal idea that ' $F$ ' is read distributively just in case ' $F a a$ ' implies ' $F a$ ' for each of $a a$.

My alternative suggestion is that ' $F$ ' is read distributively ' $F a a$ ' is true, with respect to a cover $\delta_{i}$, just in case ' $F$ ' is true of each value of $\delta_{i}(a a)$.

\section{Distributivity and Collectivity (Revised)}

(i) ' $F$ ' is read distributively iff for any $\delta_{\mathrm{i}}$, ' $F a a$ ' is true w.r.t. $\delta_{\mathrm{i}}$ only if, for any $b b$ which is a value of $\delta_{\mathrm{i}}(a a)$, ' $F b b$ ' is true w.r.t. $\delta_{\mathrm{i}}$

(ii) ' $F a a$ ' is read collectively iff it isn't read distributively

When ' $F$ ' is read distributively, we don't (necessarily) evaluate ' $F a a$ ' by considering whether ' $F$ ' is true of everything included in $a a$. Rather, we consider which pluralities $b b_{1}, \ldots, b b_{n}$ our current choice of cover maps $a a$ to, and whether ' $F$ ' is true of each of those pluralities.

On this approach, (4) comes out false when evaluated with respect to $\delta_{1}$, since $\delta_{1}$ maps the plurality $r @ h_{1} @ h_{2}$ to each of Rogers, Hart, and Hammerstein individually, and 'wrote musicals' isn't true of any of them. But (4) comes out true when evaluated with respect to $\delta_{2}$, since $\delta_{2}$ maps the plurality $r @ h_{1} @ h_{2}$ to the pluralities $r @ h_{1}$ and $r @ h_{2}$, and 'wrote musicals' is true of each of these pluralities. ${ }^{12}$

\footnotetext{
11 See Oliver and Smiley (2016: pp. 4-7) for discussion of multivalued functions.

12 Question: Instead of multi-valued functions, could we use sets? E.g. could we say that a cover $\delta_{i}$ maps $a a$ to a set, $\left\{b b_{1}, \ldots, b b_{n}\right\}$, and that ' $F a a^{\prime}$ is true w.r.t. $\delta_{\mathrm{i}}$ just in case ' $F b b_{\mathrm{i}}$ ' is true for each member of that set?

Answer: As set theory is typically understood, each member of a set is an individual. This raises a problem for (4). 'Wrote musicals' needs to distribute over Rogers and Hart, on one hand, and Rogers and Hammerstein, on the other. But while Rogers and Hammerstein can be members of a set individually, they can't be members of a set collectively (and likewise for Rogers and Hart).

We could introduce pairs, understood as individuals distinct from the individuals that make them up.
} 
On an alternative view, (4) involves a third kind of predication, distinct from both collective and distributive (Cotnoir, 2013; Landman, 1989). My view has the advantage of simplicity, since it treats the more traditional distributive reading of ' $\mathrm{Faa}$ ' from Sect. 3 as an instance of a more general phenomenon. (To recover that reading, let $\delta_{\mathrm{i}}$ map $a a$ to all and only the things included in $a a$.) So, we have reason to adopt this approach to distributive predication, independently of CAI.

\section{Plural descriptions revisited}

\subsection{An alternative analysis}

We can use this approach to distributivity to capture the intuitive application conditions of the plural description 'the $F$ ', in cases where ' $F$ ' distributes over the objects described:

\section{Plural Definite Descriptions (Revised)}

' $\gamma x x(F x x) a a$ ' is true w.r.t. $\delta_{\mathrm{i}}$ iff there's some $\delta_{\mathrm{j}}$ such that, for each $b b$ which is a value of $\delta_{\mathrm{j}}(a a)$, ' $F b b$ ' is true w.r.t. some $\delta_{\mathrm{k}}$

If there is/are some $a a$ such that ' $\gamma x x(F x x) a a$ ' is true w.r.t. $\delta_{\mathrm{i}}$, then ' $1 x x(F x x)$ ' denotes $a a$. Otherwise, ' $1 x x(F x x)$ ' denotes nothing

Clause (i) is a bit clunky, but the idea is straightforward. ' $\gamma x x(F x x) a a^{\prime}$ ' is true with respect to $\delta_{i}$ just in case there's some way of carving $a a$ into pluralities, $b b_{1}, \ldots, b b_{n}$, such that, on some (possibly distinct) way of carving things up, ' $F b b$ ' is true of all and only those pluralities. That is, let $b b_{1}, \ldots, b b_{n}$ be all the things of which ' $F$ ' is true on any cover. Then, ' $\gamma x x(F x x) a a$ ' is true with respect to $\delta_{\mathrm{i}}$ just in case some (possibly distinct) cover maps $a a$ to each of those things (and hence, $a a$ is/are collectively identical to $\left.b b_{1} @ \ldots @ b b_{n}\right)$.

' $\gamma \times x(F x x) a a$ ' is true with respect to $\delta_{i}$ only if some cover maps $a a$ to all and only the $F$ s. Whether there's such a cover is insensitive to our choice of $\delta_{\mathrm{i}}$. Thus (keeping all other indices constant), if ' $\gamma x x(F x x) a a$ ' is true with respect to any cover, it's true with respect to all of them. This ensures that the denotation of ' $1 x x(F x x)$ ', as understood in clause (ii), is also insensitive to our choice of cover, preventing awkward substitution failures.

This approach captures the intuitive application conditions of plural definite descriptions. What we want is for 'the $F \mathrm{~s}$ ' to denote the things that are $F$, and nothing else. Since ' $\gamma x x(F x x) a a '$ is true just in case $a a=b b_{1} @ \ldots @ b b_{n}$, where

Footnote 12 (continued)

Then, $\delta_{i}$ could map $a a$ to $\left.\left\{<r, h_{1}\right\rangle,\left\langle r, h_{2}\right\rangle\right\}$, and 'wrote musicals' could distribute over the members of this set. But if we already adopt the resources of plural languages, we should be skeptical of this move. Defenders of plural languages are traditionally opposed to views on which a collective predicate-like 'wrote Principia Mathematica' in 'Russell and Whitehead wrote Principia Mathematica'-is true, not of some things-i.e., Russell and Whitehead-but of some thing, typically a set with those things as members (Boolos, 1984; McKay, 2006: pp. 22-32; Oliver and Smiley, 2016: ch. 3; Yi, 2005: pp. 463-476). But if we deny that 'wrote Principia Mathematica' is true of a set, I don't see why we'd accept that 'wrote musicals' is true of a pair (so understood). 
$b b_{1}, \ldots, b b_{n}$, are the things which are $F$, we get the result that ' $1 x x(F x x)$ ' denotes those things and nothing else.

We also get the desirable result that all $F$ s are included in the $F$ s. Let $b b_{1}, \ldots, b b_{n}$ be the things which are $F$. ' $\gamma x x(F x x) a a$ ' is true just in case $a a=b b_{1} @ \ldots . . b b_{n}$. Since identity is defined as mutual inclusion, it follows that $b b_{1} @ \ldots @ b b_{n} \preccurlyeq a a$. Since ' $\leqslant$ ' is distributive at its first argument place, it follows that each of $b b_{1}, \ldots, b b_{n}$ is/are included in $a a$.

Crucially, however, we don't get the result that only $F$ s are included in the Fs. Again, letting $b b_{1}, \ldots, b b_{n}$ be the things which are $F$, if $a a$ are the $F$ s then each of $b b_{1}, \ldots, b b_{n}$ is/are included in $a a$. But $a a$ may include things which aren't $F$ s, since some $b b_{\mathrm{i}}$ included in $a a$ may themselves include non- $F \mathrm{~s} .{ }^{13}$

To see that this is a feature rather than a bug, suppose you're watching a tennis match between two two-person teams. One of these teams has Alice and Beth as its members, the other has Claire and Diane as its members. Now consider the plural description, 'the teams'. What does it denote? It seems to me that it denotes the four people: Alice, Beth, Claire, and Diane. (Each two-person team, it seems to me, just is the people that make it up.) But this is inconsistent with the analysis of plural descriptions from Sect. 3. On that analysis, if 'the $F$ s' denotes $x x$, then only $F$ s are included in $x x$; so, 'the teams' can't denote the four people, since none of them is a team. By contrast, it's perfectly consistent with my analysis. On my analysis, all that's required for 'the teams' to denote the four people is that there be a cover which maps them to those pluralities $b b_{1} \ldots b b_{n}$ such that 'is/are a team' is true of all and only those pluralities. And there is such a cover, namely that which maps them to the pairs Alice@Beth and to Claire@Diane. ${ }^{14}$

This argument generalizes. We can introduce a plural description, 'the $F \mathrm{~s}$ ', in cases where ' $F$ ' distributes over things in the plurality (i.e. 'the $F$ s' doesn't mean 'the things which are collectively $F$ '), and yet ' $F$ ' applies only to (proper) pluralities. That is, when we group together the $F \mathrm{~s}$, we may be grouping pluralities together into a larger plurality. But then, since each $F$-plurality may include non- $F$ s (e.g. a team includes people who aren't teams), this larger plurality of $F$-pluralities may also include non- $F$ s. According to the original analysis, 'the $F$ s' can't denote such a plurality. But since it apparently can (e.g. 'the teams' can denote the members of the teams), the original analysis should be rejected in favour of one, like mine, which secures this result. ${ }^{15}$

\footnotetext{
13 There's still a sense in which nothing 'other than' $F$ s are included in the $F$ s. If $b b_{1}, \ldots, b b_{n}$ are all the $F$ s, then nothing outside $b b_{1} @ \ldots @ b b_{n}$ gets included in the $F$ s. Thus, we can circumscribe the things which are $F$ and use 'the $F$ s' to refer to that plurality and nothing outside it.

14 Objection: Alice and Beth don't themselves exemplify the property being a team. Rather, they constitute an individual which does exemplify that property.

Reply: This view has been argued against elsewhere - see, e.g., Horden and López de Sa (2020) and Payton (2019: pp. 14-18). Here, I assume it's false.

15 On an alternative approach, talk about 'pluralities of pluralities' is captured using 'higher-level' plural terms and variables, which stand to ordinary plural ones as the latter stand to singular ones (Linnebo and Nicolas, 2008; Rayo, 2006). However, such 'higher-level' plural resources are highly controversial (Ben-Yami, 2013; McKay, 2006: pp. 137-139; Uzquiano, 2004: pp. 438-440), and my approach doesn’t require them.
} 


\subsection{The tension resolved}

This analysis of plural descriptions resolves the tension with CAI.

According to CAI, if a wall fuses both the bricks from which it's composed and the atoms from which the bricks are composed, then the bricks are the atoms. But given the analysis of plural descriptions from Sect. 3, that identity claim is false: the bricks include all and only bricks, while the atoms include all and only atoms; no plurality can satisfy both conditions.

By contrast, on my analysis, while 'the bricks' denotes a plurality which includes all the bricks, that plurality needn't include only bricks, so nothing prevents an atom from being included in the bricks. Likewise, the plurality denoted by 'the atoms' includes all the atoms, but needn't include only atoms, so nothing prevents a brick from being included in the atoms.

Putting the point in positive terms, what's required for the bricks to be identical to the atoms (and hence for 'the bricks' and 'the atoms' to be co-denoting) is that there are some $a a$ such that (i) some cover $\delta_{\mathrm{i}}$ maps $a a$ to all and only the bricks, $b b_{1}, \ldots, b b_{n}$ (so $a a=b b_{1} @ \ldots @ b b_{n}$ ), while (ii) some cover $\delta_{\mathrm{j}}$ maps $a a$ to all and only the atoms, $c c_{1}, \ldots, c c_{n}$ (so $a a=c c_{1} @ \ldots @ c c_{n}$, and by the transitivity of identity, $\left.b b_{1} @ \ldots @ b b_{n}=c c_{1} @ \ldots @ c c_{n}\right)$. Nothing prevents both conditions from being satisfied, so there's no reason to think that the atoms and the bricks must be distinct.

\section{Comprehension revisited}

\subsection{An alternative schema}

Finally, with my alternative analysis of plural descriptions in place, we can adopt an alternative comprehension schema.

The intuitive idea behind Comprehension, recall, is that if there's at least one $\varphi$-er, then there must be such things as the $\varphi$-ers: as long as there's at least one $\varphi$-er, we should be able to single them all out as a plurality. Comprehension cashes this out using the original analysis of 'the $\varphi$-ers', on which it denotes a plurality which includes all and only the $\varphi$-ers. Since we have reason to prefer an alternative analysis of plural descriptions, we already have reason to prefer a comprehension schema which uses it.

There's a further reason to be dissatisfied with Comprehension, and to prefer a schema which uses my analysis of plural descriptions. The antecedent of Comprehension is ' $\exists x \varphi x$ ': it holds only when ' $\varphi$ ' is true of some individual. But we should want a schema which holds when ' $\varphi$ ' is collectively satisfied by a (proper) plurality. First, an analogue of the intuitive thought behind Comprehension still holds, in such cases-e.g. if there's at least one team, then we ought to be able (in principle) to single out the teams as a plurality; there ought to be such a plurality as the teams, or the pluralities which are teams. Second, Comprehension is supposed to help us give a semantics for higher-order languages on which the denotation of a predicate is just the things which satisfy it. And such a semantics is only complete if it applies to predicates collectively satisfied by (proper) pluralities. 
A straightforward pluralization of Comprehension, which retains the original analysis of plural descriptions, won't work for these purposes.

\section{Comprehension 2}

$\exists x x \varphi x x \supset \exists y y \forall z z(z z \leqslant y y \equiv \phi z z)$

For, this schema has a false instance:

$$
\exists x x T x \supset \supset \exists y y \forall z z(z z \leqslant y y \equiv T z z)
$$

Alice and Beth are a team, so it's true that some $x x$ are a team. But it's false that there's a plurality which includes all and only teams: since Alice and Beth are a team, any plurality which includes all the teams must include Alice and Beth collectively, and hence individually; but then, this plurality doesn't include only teams, since neither Alice nor Beth is a team. ${ }^{16}$

If we adopt my analysis of plural descriptions, we can do better:

\section{Comprehension 3}

If ' $\exists x x \phi x x$ ' is true w.r.t. $\delta_{\mathrm{i}}$, then ' $\exists y y(\gamma z z(\varphi z z) y y)$ ' is true w.r.t. $\delta_{\mathrm{i}}$

Given my analysis, Comprehension 3 expresses the intuitive thought that, if there's at least one $\varphi$-er, then there are the $\varphi$-ers: if some thing(s) is/are (collectively) $\varphi$, then there are some things, $a a$, and some cover, $\delta_{\mathrm{j}}$, such that $\delta_{\mathrm{j}}$ maps $a a$ to some $b b_{1}, \ldots, b b_{n}$, where $b b_{1}, \ldots, b b_{n}$ are all and only the $\varphi$-ers.

Now, instead of (5) we get:

$$
\exists x x T x \supset \exists y y(\gamma z z(T z z) y y)
$$

Alice and Beth are a team, so it's true that some $x x$ are a team. By $\left(5^{*}\right)$, some $y y$ satisfy the predicate 'are the teams' —or, ' $\gamma z z(T z z)$ '. That is, for some $a a$ and $\delta_{\mathrm{i}}$, the values of $\delta_{\mathrm{i}}(a a)$ are all and only the teams $b b_{1}, \ldots, b b_{n}$, and so $a a=b b_{1} @ \ldots @ b b_{n}$. Since Alice and Beth are a team, they're a value of $\delta_{\mathrm{i}}(a a)$, and they're included in $b b_{1} @ \ldots @ b b_{n}$. But given my analysis of plural descriptions, that's consistent with $b b_{1} @ \ldots @ b b_{n}$ (and hence $a a$ ) being the denotation of 'the teams'. So, we can say that there are such things as the teams (namely, $b b_{1} @ . . . @ b b_{n}$ ), and let this plurality serve as the semantic value of ' $T$. Thus, we have good reason to adopt Comprehension 3 independent of CAI. ${ }^{17}$

\subsection{The tension resolved}

Comprehension 3 resolves the tension with CAI.

\footnotetext{
${ }_{16}$ Remember, plural variables are inclusive, so each of Alice and Beth can be a value for ' $z z$ '.

17 Note: since plural variables are inclusive, Comprehension 3 still holds when ' $\varphi$ ' is true only of individuals.
} 
According to Comprehension, if there's at least one brick, there's a plurality which includes all and only bricks. This is meant (given the analysis of plural descriptions from Sect. 3) to capture the thought that, if there's at least one brick, there are the bricks. But according to CAI, there can be no such plurality: any plurality which includes a brick will also include the atoms from which it's composed. So, CAI conflicts with Comprehension: the existence of a brick doesn't imply the existence of the bricks.

By contrast, Comprehension 3 is consistent with CAI. It's still true that any plurality which includes a brick will also include the atoms from which it's composed. But since it's no longer required that the bricks include only bricks, this poses no problem for the intuitive thought that, given the existence of at least one brick, there must be such things as the bricks. For, there can still be some $a a$ and some cover $\delta_{\mathrm{i}}$ such that $\delta_{i}$ maps $a a$ to all and only bricks. These $a a$ can serve as the denotation of 'the bricks' with respect to any cover, and so we can preserve the intuitive thought that, given the existence of a brick, there are such things as the bricks.

\section{Summing up}

In Sect. 3, we used standard resources of plural languages, including especially a formal treatment of the distributive/collective distinction, to develop (i) a formal analysis of distributive plural descriptions and (ii) a comprehension schema making use of that analysis. That analysis, and that schema, were in tension with CAI.

I've developed an alternative treatment of distributivity and collectivity, an alternative analysis of plural descriptions, and an alternative comprehension schema. I've argued that we have independent reason to adopt each component, and shown that the resulting package is consistent with CAI. Thus, a defender of CAI needn't deny that ordinary plural descriptions like 'the bricks' refer (since she needn't think that 'the bricks' denotes a plurality which includes only bricks), nor reject the intuitive idea behind plural comprehension (since she can allow that 'the bricks' refers, and hence that there are the bricks). We can identify wholes with their parts, and still have all the pluralities we could want. ${ }^{18}$

\section{Alternatives}

Extant responses to the problems from Sect. 3 typically involve rejecting CAI, as I've understood it, or accepting that there are fewer pluralities than we ordinarily think there are. Here, I'll briefly compare my solution to three such alternatives.

\footnotetext{
18 Objection: Your approach doesn't get us all the pluralities we could want. E.g., there's no plurality which includes all and only the bricks.

Reply: All we should want, or expect, is for there to be such a plurality as the bricks. What's pre-theoretically clear is that we can (in principle) single out the bricks. What's not pre-theoretically clear is that doing so requires that we single out a plurality which includes all and only bricks (and I've argued in Sect. 5.1 that this assumption about how plural descriptions work is false, for reasons independent of CAI).
} 


\subsection{Sider}

Sider (2014) recommends that the defender of CAI bite the bullet and accept that ordinary descriptions like 'the bricks' don't refer, and that the intuitive thought behind Comprehension is false. He nonetheless recommends a new comprehension schema which he thinks will suit the defender's purposes.

First, Sider introduces a schematic notion of 'fusion', or 'S-fusion' (2014: p. 214):

\section{Definition of 'S-Fu'}

$S-F u(a, \phi) \equiv_{\mathrm{df}} \forall x(\phi x \supset x \leq a \& \forall y(y \leq a \supset \exists z(\varphi z \& y \circ z))$

$a \mathrm{~S}$-fuses the individual $\varphi$-ers (which is not to say that $a$ fuses the $\varphi$-ers; see below) just in case each $\varphi$-er is part of $a$, and each part of $a$ overlaps a $\varphi$-er. ${ }^{19}$ Sider then formulates his comprehension schema:

\section{Comprehension (Sider)}

$\exists x \phi x \supset \exists x x \exists y(S-F u(y, \varphi) \& \forall z(z \leqslant x x \equiv z \leq y))$

If there's at least one $\varphi$-er, then there exists (i) an $S$-fusion of the individual $\varphi$-ers, and (ii) a corresponding plurality, $x x$, which include all and only parts of that S-fusion. Notice, this doesn't imply that there are some $x x$ which include all and only $\varphi$-ers. Given that there's at least one brick, there's an S-fusion of the individual bricks (i.e. an object which has all the bricks as parts, and all of whose parts overlap at least one brick), and a plurality which includes all and only the parts of that S-fusion. But this plurality can include things other than bricks; e.g. it will include all the atoms that are parts of the bricks (and hence parts of the S-fusion of the individual bricks).

Sider's comprehension schema gets a similar result to my Comprehension 3: given the existence of a $\varphi$-er, we're no longer committed to a plurality which includes all and only the individual $\varphi$-ers. But Comprehension $\mathbf{3}$ is motivated by my analysis of plural descriptions from Sect. 5. Given that analysis, Comprehension 3 says that, given the existence of a $\varphi$-er, there are the $\varphi$-ers. By contrast, Sider (2014: pp. 215-216) retains the original analysis of plural descriptions from Sect. 3, so the $x x$ quantified over in his comprehension schema aren't (necessarily) the $\varphi$-ers, by his lights.

Of course, we could develop an analysis of plural descriptions to go with Sider's comprehension schema. We could claim that 'the $F \mathrm{~s}$ ' denotes the plurality corresponding to the S-Fusion of the individual $F \mathrm{~s}$ :

\section{Plural Definite Descriptions (Sideresque)}

(i)

$$
\text { ' } \gamma x x(F x x) a a \text { ' is true iff ' } \forall y y\left(y y \leqslant a a \equiv y y \leq u x(S-F u(x, F)) \text { ' is true } e^{20}\right.
$$

\footnotetext{
${ }^{19}$ Sider (2014: pp. 214-215). To derive an equivalent of the original definition of ' $F u(a, b b)$ ', substitute ' $\lambda x .(x \leqslant b b)$ ' for ' $\varphi$ '.

${ }^{20}$ In keeping with CAI, I allow that many $y y$ can collectively be a part of $x$.
} 
(ii) If there is/are some $a a$ such that $\gamma x x(F x x) a a$, then ' $1 x x(F x x)$ ' denotes $a a$. Otherwise, ' $x x(F x x)$ ' denotes nothing

But this analysis causes trouble if distributive predication is understood as in Sect. 3.

Consider (6):

The writers of Principia Mathematica are men

According to the Sideresque analysis, 'the writers of Principia Mathematica' denotes those $x x$ which include all and only the parts of the S-fusion of writers of Principia Mathematica-i.e. it denotes those $x x$ which include Russell, Whitehead, all of their proper parts, and any sums of these things there may be. Given the approach to distributive predication from Sect. 3, 'are men' is true of $x x$ just in case everything included in $x x$ is a man. But then (6) is false, since $x x$ includes, e.g., all of Russell's atomic parts, none of which is a man. We could solve this problem by adopting an alternative approach to distributive predication, but it's not clear what this alternative could be. ${ }^{21}$

My view doesn't have this problem. The approach to distributive predication from Sect. 4 shows how (6) can be true, given CAI. Let $\delta_{1}$ be a cover which maps $r @ w$ just to Russell and to Whitehead: $\delta_{1}(r @ w)=r, w$. Relative to $\delta_{1},(6)$ is true just in case each of Russell and Whitehead was man, so the intuitive truth-conditions of (6) are preserved. But (6) can still be true, relative to $\delta_{1}$, even if $r @ w$ includes some things which aren't men. What matters for the truth of (6), relative to $\delta_{1}$, isn't whether 'are men' is true of each thing included in $r @ w$, but whether it's true of each value of $\delta_{1}(r @ w)$. My view thus gives the defender of CAI a satisfying treatment of distributive predication and plural descriptions, and plural comprehension.

\subsection{Loss}

Loss (2019) uses Sider's notion of 'S-fusion' to provide another alternative comprehension schema, but he's more restrictive about what gets included in the plurality corresponding to an S-Fusion. Loss assumes that every individual is either an atom or the fusion of some atoms, and takes this to suggest the following schema:

\section{Comprehension (Loss)}

$\exists x \varphi x \supset \exists x x \exists y(S-F u(y, \varphi) \& \forall z(z \leqslant x x \equiv z \leq y \& \operatorname{Atom}(z))$

If there's at least one $\varphi$-er, then there exists (i) an $S$-fusion of the individual $\varphi$-ers, and (ii) some $x x$ which include all and only the atomic parts of that S-fusion (11). As with Sider's schema (and mine), this doesn't imply that there are some $x x$ which include all and only $\phi$-ers: the S-Fusion of the bricks will have some atoms as proper

\footnotetext{
21 Sider (2007: p. 58) is well aware of the problems with combining CAI and the approach to distributivity from Sect. 3. I suspect he would reject the Sideresque analysis for that reason.
} 
parts, so from the fact that there's at least one brick, it doesn't follow that any plurality includes all and only bricks.

Unlike with Sider's schema (and mine), there isn't even guaranteed to be a plurality which includes any $\phi$-ers. The plurality corresponding to the S-Fusion of the bricks includes all and only the atomic parts of that S-Fusion, i.e. the atoms which make up the individual bricks. Since no brick is an atom, no brick is included in this plurality. Indeed, when combined with CAI, Loss's schema entails that the only pluralities there are, are pluralities of atoms (2019: p. 11).

It may seem that Loss's proposal, like Sider's, requires us to think that there are fewer pluralities than we'd ordinarily expect. However, Loss has a way of avoiding this result.

Following Sider (2011), Loss (2020) claims that some quantifiers are more 'metaphysically perspicuous' than others: they do a better job at 'carving nature at the joints'. Moreover, he suggests, the most joint-carving quantifiers (singular and plural) range only over atoms. Thus, Loss's comprehension schema is true if we read ' $\exists x x$ ' as using the most joint-carving plural quantifier: in the most joint-carving sense of 'there are', there are only pluralities of atoms. That's consistent, however, with there being less joint-carving plural quantifiers which range over composite objects. It may be that there are such pluralities as the bricks, even if only in a less joint-carving sense of 'there are' (2020: p. 9).

This package of views comes at a high metametaphysical cost: we must accept, not only that reality has 'quantificational structure', ${ }^{22}$ but that the most joint-carving quantifiers range only over atoms. By contrast, my approach is motivated by linguistic evidence which is independent of any metametaphysical claims about quantificational structure.

\subsection{Cotnoir}

In Sect. 4 I claimed that whether a plural predication is true depends on how we 'carve up' reality. Cotnoir (2013), following Baxter (1988), claims that what exists (i.e. what can be quantified over) also depends on how we 'carve up' reality. E.g., we can carve a single portion of reality into one thing, a six-pack of orange juice, or into many things, the six individual juices. The question of what exists (The sixpack? The six individual juices?) has no answer, independently of which carving we choose (Baxter, 1988: pp. 200-201; 210-211).

Moreover, on this view, while we can 'carve up' a portion of reality into one thing or many (e.g. a single whole and its many parts) we can't do both at once. No acceptable domain of quantification includes both a whole and its parts. Thus, we can never say that a whole is identical to its parts, and so CAI, as I've stated it, is false. Nonetheless, a whole and its parts stand in a kind of identity relation: they're the same portion of reality. Cotnoir calls this relation 'general identity'.

${ }^{22}$ See, e.g., Hirsch (2013), Thomasson (2014: pp. 308-317), and Warren (2016) for concerns. 
To see how this works, assume that a portion of reality is simply some atoms, $x x$. We can carve these atoms up in different ways, e.g. into a single whole, $a$, or into the parts of that whole, $b b$, at a certain level of composition. ${ }^{23}$ Since we can't adopt both carvings at once, we can't have both $a$ and $b b$ in the domain of quantification, so ' $a=b b$ ' is unstateable. What we $c a n$ do, however, is adopt a domain of quantification that includes just the uncarved atoms and say, from this new perspective, that the terms ' $a$ ' and ' $b b$ ' denote the same portion of reality, the same collection of atoms (Cotnoir, 2013: pp. 301-306).

The problems with plural descriptions and Comprehension from Sect. 3 don't arise, on this view. Since we can never have both a brick and its constituent atoms in the domain of quantification, we can never have both the bricks and the atoms in the domain of quantification. Thus, we can't say that these pluralities are identical. But then, we can adopt the analysis of plural descriptions from Sect. 3: the bricks (which can only be in the domain if the atoms aren't) include all and only bricks, and the atoms (which can only be in the domain if the bricks aren't) include all and only atoms. Likewise, while comprehension is, in a sense, restricted (2013: 303), we can accept Comprehension as stated: given the existence of a brick, there's a plurality which includes all and only bricks (since no plurality which includes a brick can also include its constituent atoms).

I take my view to be better motivated. We've seen the linguistic evidence for my approaches to distributive predication, plural descriptions, and plural comprehension, and that these approaches are consistent with CAI. Is there reason to reject CAI in favour of Cotnoir's 'general identity' view?

Cotnoir rejects CAI on the ground that, if a whole is identical to its many parts, then the very same thing(s) is/are both one and many, which is impossible (2013: 301). This argument also motivates his claims that what exists is relative to a carving, and that we can't carve a portion of reality into one thing and many at once, since these claims allow us to avoid seemingly inconsistent number-ascriptions. ${ }^{24}$ But I've argued elsewhere (Payton, 2019; n.d.) for a semantics of number ascriptions which renders ' $a$ is one thing' and ' $a$ is many things' consistent, and which does so without appeal to discrete domains of quantification or the notion of 'general identity'. ${ }^{25}$

\section{Nihilism}

Calosi (2016: pp. 225-228) and Loss (2018: pp. 371-372) argue that CAI leads to compositional nihilism: composition never occurs; everything is a mereological atom, whose only part is itself.

\footnotetext{
${ }^{23}$ Since parthood is transitive, we can't always carve a whole into all its parts at once. E.g., we can't have both the bricks which compose the wall and the atoms which compose the bricks in our domain of quantification.

24 See also Baxter (1988: p. 193).

25 For further discussion of Cotnoir's view, see Carrara and Lando (2016) and Hawley (2013).
} 


\section{Nihilism}

$\forall x \forall y(y \leq x \equiv y=x)$

Nihilism renders CAI completely uninteresting: 'Composite objects would be identical to their parts, if there were any, which there aren't.' It also undermines my claim that we can accept CAI while believing in such pluralities as the bricks. (If Nihilism is true, there aren't any bricks.)

Fortunately, my approach to plural descriptions and my comprehension schema block these arguments.

\subsection{Calosi}

CAI implies Collapse (Sider, 2007: pp. 57-58; 2014: 213):

\section{Collapse}

$\forall x \forall y y(F u(x, y y) \supset \forall z(z \leq x \equiv z \leqslant y y))$

If $x$ fuses $y y$, then $z$ is part of $x$ just in case $z$ is included in $y y$. Calosi argues that, if Collapse is true, every object is indistinguishable from each of its parts (i.e. if $b$ is part of $a$, then $a$ and $b$ have all the same properties). But if some $b$ is a proper part of $a$, they have different properties (e.g. $a$ has itself as a part, but $b$ doesn't have $a$ as a part). So, if Collapse is true, nothing has proper parts; Nihilism is true (2016: 227-228).

Calosi's argument comes in two parts: first, if $b$ is part of $a$, then (given Collapse) there's no property which $a$ has but $b$ lacks; second if $b$ is part of $a$, then (given Collapse) there's no property which $b$ has but $a$ lacks. I'll only address the first part here. The second part is structurally similar to the first, and fails for the same reason.

Suppose that $a$ fuses $b b$, which includes $b(\mathrm{C} 1)$. By Collapse, $b$ is part of $a(\mathrm{C} 2)$. Now suppose for reductio that $a$ has some property, call it ' $P$ ', which $b$ lacks (C3), and let ' $c c$ ' denote a plurality which includes all and only the ' $P$-parts' of $a$, i.e., the parts of $a$ which possesses $P$ (C4). By the definition of 'fusion', $a$ fuses $c c$ (C5). ${ }^{26}$ But since $b$ is part of $a, b$ is included in $c c$ by Collapse (C6). By C4, $b$ a $P$-part of $a$ (C7), so $b$ possesses $P(\mathrm{C} 8)$, contradicting our assumption (C9).

$\begin{array}{lll}\text { (C1) } & F u(a, b b) \& b \leqslant b b & \text { [Assumption] } \\ \text { (C2) } & \therefore b \leq a & \text { [C1, Collapse] } \\ \text { (C3) } & P a \& \sim P b & \text { [Assumption] } \\ \text { (C4) } & \forall x(x \leqslant c c \equiv(x \leq a \& P x)) & \text { [Premise] } \\ \text { (C5) } & \therefore F u(a, c c) & \text { [C3, C4, definition of ' } F u \text { '] } \\ \text { (C6) } & \therefore b \leqslant c c & \text { [C2, C5, Collapse] }\end{array}$

\footnotetext{
${ }^{26}$ Proof: First, $\forall x(x \leqslant c c \supset x \leq a)$ follows trivially from C4. Second, we have $\forall y(y \leq a \supset \exists z(z \leqslant c c \& y$ 。 $z)$ ): since we've assumed $P a$, and parthood is reflexive, we have ' $a \leq a \& P a$ '; by $\mathrm{C} 4, a \leqslant c c$; but then, every part of $a$ overlaps one of $a$ 's $P$-parts, since every part of $a$ overlaps $a$.
} 


$\begin{array}{lll}\text { (C7) } & \therefore b \leq a \& P b & {[\mathrm{C} 6, \mathrm{C} 4]} \\ \text { (C8) } & \therefore P b & {[\mathrm{C} 7]} \\ \text { (C9) } & \perp & {[\mathrm{C} 3, \mathrm{C} 8]}\end{array}$

By reductio, if $b$ is part of $a$, there's no property which $a$ has and $b$ lacks (2016: 226-227).

The natural reply, for a defender of CAI, is to reject C4, the premise that there's a plurality which includes all and only the $P$-parts of $a$. In particular, she should reject the left-to-right direction (i.e., that $c c$ includes only $P$-parts of $a$ ) since that's what licenses the move from $\mathrm{C} 3$ to $\mathrm{C} 5$, and the move from $\mathrm{C} 6$ to $\mathrm{C} 7$.

The intuitive motivation for $\mathrm{C} 4$ is that, given that the notion of a $P$-part of $a$ is well-defined, we're free to introduce a plural term, ' $c c$ ' which behaves like 'the $P$-parts of $a$ '. Given the analysis of plural descriptions from Sect. 3, the denotation of ' $c c$ ' must include all and only the $P$-parts of $a$-hence C4.

But on my analysis of plural descriptions, while the denotation of ' $c c$ ' must include all the $P$-parts, it needn't include only the $P$-parts. All that's required for ' $c c$ ' to behave like 'the $P$-parts of $a$ ' is that some cover maps $c c$ to all and only the $P$-parts. Equivalently: it requires only that ' $c c$ ' denote some $b b_{1}, \ldots, b b_{n}$ such that (i) each of them is identical to one of $a$ 's $P$-parts, and (ii) each $P$-part of $a$ is identical to one of them. That's consistent with there being some things included in $c c$ which aren't $P$-parts. (Just as, on my view, the bricks include some atoms.)

Thus, on my analysis, the left-to-right direction of $\mathrm{C} 4$ is unmotivated; there's no reason to think that any plurality includes only the P-parts of $a$. But then, the moves to $\mathrm{C} 5$ and $\mathrm{C} 7$ are blocked. The reductio, and hence the argument for Nihilism, fails.

\subsection{Loss}

Loss's first argument (2018: 371) appeals to Calosi's notion of an improper plurality, i.e. a plurality which includes only one thing. Say that $a a$ is the improper plurality of $a$-or ' $I P(a a, a)$ ' - just in case $a$ is the only thing included in $a a$. Now let ' $a$ ' denote some arbitrary object and ' $a a$ ' its improper plurality (L1). By the definition of 'fusion', $a$ fuses $a a$ (L2), ${ }^{27}$ so by Collapse, $a a$ includes all and only the parts of $a$ (L3). But the only thing included in $a a$ is $a$ (L4), so the only part $a$ has is itself (L5). Generalizing, the only part anything has is itself; that is, Nihilism is true (L6).

$\begin{array}{lll}\text { (L1) } & I P(a a, a) & \text { [Assumption] } \\ \text { (L2) } & \therefore F u(a, a a) & \text { [L1, definition of ' } F u^{\prime} \text { ] } \\ \text { (L3) } & \therefore \forall x(x \leqslant a a \equiv x \leq a) & \text { [L2, Collapse] } \\ \text { (L4) } & \therefore \forall x(x \leqslant a a \equiv x=a) & \text { [L1] } \\ \text { (L5) } & \therefore \forall x(x \leq a \equiv x=a) & \text { [L3, L4] } \\ \text { (L6) } & \therefore \forall x \forall y(y \leq x \equiv y=x) & \text { [L5, } \forall \text {-int] }\end{array}$

\footnotetext{
${ }^{27}$ Proof: First, we have $\forall x(x \leqslant a a \supset x \leq a)$, since the only thing included in $a a$ is $a$ itself, and parthood is reflexive. Second, we have $\forall y(y \leq a \supset \exists z(z \leqslant a a \& y \circ z))$ : by the definition of 'overlap' (n. 4), any part of $a$ overlaps $a$. Since $a$ is the only thing included in $a a$, it follows that any part of $a$ overlaps one of those things, namely $a$.
} 
This argument assumes that any arbitrary object has an improper plurality. (That's what licenses L1, and the generalization from L5 to L6.) The natural reply, for a defender of CAI, is that this assumption is false. In particular, composite objects don't have improper pluralities.

If $a$ is a composite object, then by CAI it's identical to all its parts collectively, and hence includes each of them individually. But then, $a$ can't have an improper plurality: any plurality which includes $a$ will thereby include all of $a$ 's proper parts, each of which is distinct from $a$. If CAI is true, the only objects which have improper pluralities are mereological atoms. L1 is licensed only if $a$ is a mereological atom, and L5 only generalizes to such things. But then, the conclusion is trivial: all mereological atoms are mereological atoms. ${ }^{28}$

This reply might seem cheap: 'Your modus ponens is my modus tollens'. But the claim that composite objects don't have improper pluralities is part and parcel of CAI: it's neither a surprising bullet to bite, nor an ad hoc claim wheeled in just to block Loss's argument.

Moreover, the only clear reason to assume that any arbitrary object must have an improper plurality, is that this assumption is an instance of Comprehension:

$$
\exists x(x=a) \supset \exists x x \forall y(y \leqslant x x \equiv(y=a))
$$

(If $a$ exists, then there's a plurality which includes all and only individuals identical to $a$, i.e. an improper plurality of $a$.) But if we replace Comprehension with Comprehension 3, as I've argued we should, we don't get (7). Instead, we get:

$$
\text { If ' } \exists x x(x x=a) \text { ' is true w.r.t. } \delta_{\mathrm{i}} \text {, then ' } \exists y y(\gamma z z(z z=a) y y) \text { ' is true w.r.t. } \delta_{\mathrm{i}}
$$

If anything is identical to $a$, then there's a plurality $a a$ and a cover $\delta_{\mathrm{j}}$ such that $\delta_{\mathrm{j}}$ maps $a a$ to some $b b_{1}, \ldots, b b_{n}$, where $b b_{1}, \ldots, b b_{n}$ are the things which are identical to $a$. Of course, there's only one such thing, namely $a$ itself, so $\delta_{\mathrm{j}}(a a)=a$. But that's consistent with there being two or more things included in $a a$, each of which is distinct from $a$. aa may be a proper plurality of things, $b b_{1} @ \ldots @ b b_{n}$, such that $\delta_{\mathrm{j}}\left(b b_{1} @ \ldots @ b b_{n}\right)=a$.

Loss's second argument (2018: 372) appeals to a plausible mereological principle:

\section{Weak Company}

$\forall x \forall y(x<y \supset \exists z(z<y \& z \neq y))$

If an object has at least one proper part, then it has at least two. Now, assume that Nihilism is false, i.e., there's at least one object with a proper part (L7), and let ' $a$ ' and ' $b$ ' denote, respectively, this object and one of its proper parts (L8). Let ' $c c$ ' denote some things such that the individuals included in $c c$ are all and only things

\footnotetext{
${ }^{28}$ Loss (2018: pp. 372-373) considers a different kind of skeptic about improper pluralities, who grants that everything has an improper plurality, but rejects the inference from L1 to L2. By contrast, I reject L1 outright. See Loss (2019: p. 10) for his own way of blocking the road to Nihilism.
} 
identical either to $a$ or to $b$ (L9). By the definition of 'fusion', $a$ fuses $c c$ (L10) ${ }^{29}$ By Collapse, every part of $a$ is included in $c c$ (L11) and is therefore identical to either $a$ or $b$ (L12). Now, by the definition of 'proper part', $x$ is a proper part of $a$ only if it's distinct from $a$. So, by L12, any proper part of $a$ must be identical to $b$ (L13). Therefore, $a$ has only one proper part, namely $b$ (L14).

$$
\begin{aligned}
& \exists x \exists y(y<x) \\
& \therefore b<a \\
& \forall x(x \leqslant c c \equiv(x=a \vee x=b)) \\
& \therefore F u(a, c c) \\
& \therefore \forall x(x \leq a \supset x \leqslant c c) \\
& \therefore \forall x(x \leq a \supset(x=a \vee x=b)) \\
& \therefore \forall x(x<a \supset x=b) \\
& \therefore \exists y \forall x(x<a \supset x=y)
\end{aligned}
$$

[Assumption]

[L7, $\exists$-elim $\times 2]$

[Premise]

[L8, L9, definition of ' $F u$ ']

[L10, Collapse $]$

[L9, L11]

[L12, definition of ' $<$ ']

[L13, ヨ-int]

This contradicts Weak Company. By reductio, L7 is false and Nihilism is true.

The natural reply is to reject L9, the premise that there's a plurality the only individuals included in which are $a$ and $b$. If we assume that $b$ is a proper part of $a$, then by Weak Company, there are some individuals $c_{1} \ldots c_{n}$ such that each of them is a proper part of $a$ and each of them is distinct from $b$ (there may, of course, only be one such individual). By CAI, $a$ is identical to $b @ c_{1} @ \ldots @ c_{n}$, and so it includes each of $c_{1} \ldots c_{n}$. But then, no plurality meets the condition laid down in L9: any plurality which includes $a$ includes each of $c_{1} \ldots c_{n}$, each of which is distinct both from $a$ and from $b$.

Again, this reply isn't as cheap as it might seem. Granting Weak Company, the rejection of L9 is neither a surprising bullet to bite nor an ad hoc maneuver, but simply part and parcel of CAI. And the only clear reason for thinking that there must be a plurality which satisfies L9 is that this is simply an instance of Comprehension:

$$
\exists x(x=a \vee x=b) \supset \exists x x \forall y(y \leqslant x x \equiv(y=a \vee y=b))
$$

(If $a$ and $b$ exist, there's a plurality which includes all and only individuals identical to either $a$ or $b$.) But given Comprehension 3 we only get:

\footnotetext{
29 Proof: First, we have $\forall x(x \leqslant c c \supset x \leq a)$ : by L9, the only things included in $c c$ are $a$ and $b$, and $a$ is part $a$ by reflexivity while $b$ is part of $a$ by L8. Second, we have $\forall y(y \leq a \supset \exists z(z \preccurlyeq c c \& y \circ z))$ : since $a$ is included in $c c$, every part of $a$ overlaps something included in $c c$, namely $a$.
} 


$$
\text { If ' } \exists x x(x x=a \vee x x=b) \text { ' is true w.r.t. } \delta_{\mathrm{i}} \text {, then ' } \exists y y(\gamma z z(z z=a \vee z z=b) y y) \text { ' is true w.r.t. } \delta_{\mathrm{i}}
$$

If anything is identical either to $a$ or to $b$, then there's a plurality $a a$ and a cover $\delta_{\mathrm{j}}$ such that $\delta_{\mathrm{j}}$ maps $a a$ to some $b b_{1}, \ldots, b b_{n}$, where $b b_{1}, \ldots, b b_{n}$ are the things which are identical either to $a$ or to $b$. Of course, there are only two such things, namely $a$ and $b$ themselves, so $\delta_{\mathrm{j}}(a a)=a, b$. This entails that $a a=a @ b$, so each of $a$ and $b$ is included in $a a$. But that's consistent with there being other individuals included in $a a^{30}$

Thus, my analysis of plural descriptions and my comprehension schema block the road from CAI to Nihilism. ${ }^{31}$

Acknowledgements Thanks to Noa Latham, David Liebesman, and three anonymous referees for feedback on earlier versions of this paper. Research was funded by a Postdoctoral Fellowship from the Social Sciences and Humanities Research Council of Canada.

\section{References}

Baxter, D. M. (1988). Many-one identity. Philosophical Papers, 17(3), 193-216

Ben-Yami, H. (2013). Higher-level plurals vs articulated reference, and an elaboration of salva veritate. Dialectica, 67(1), 81-102

Bohn, E. D. (2014). Unrestricted composition as identity. In A. J. Cotnoir \& D. M. Baxter (Eds.), Composition as identity. (pp. 143-165). New York: Oxford University Press.

Boolos, G. (1984). To be is to be the value of a variable (or to be the values of some variables). Journal of Philosophy, 81(8), 430-449

Calosi, C. (2018). Failure or boredom: The pendulum of composition as identity. American Philosophical Quarterly, 55(3), 281-291

Calosi, C. (2016). Composition as identity and mereological nihilism. Philosophical Quarterly, 66(263), 219-235

Carrara, M., \& Lando, G. (2017). Composition and relative counting. Dialectica, 71(4), 489-529

Carrara, M., \& Lando, G. (2016). Composition, indiscernibility, coreferentiality. Erkenntnis, 81(1), $119-142$

Cotnoir, A. J. (2013). Composition as general identity. In K. Bennett \& D. W. Zimmerman (Eds.), Oxford studies in metaphysics. (Vol. 8, pp. 294-322). Oxford University Press.

Gillon, B. S. (1992). Towards a common semantics for English count and mass nouns. Linguistics and Philosophy, 15(6), 597-639

Hawley, K. (2013). Cut the pie any way you like? Cotnoir on general identity. In K. Bennett \& D. W. Zimmerman (Eds.), Oxford studies in metaphysics. (Vol. 8, pp. 323-330). Oxford University Press.

Hirsch, E. (2013). The metaphysically best language. Philosophy and Phenomenological Research, 87(3), 709-716

Horden, J., \& Lópezde Sa, D. (2020). Groups as pluralities. Synthese. https://doi.org/10.1007/ s11229-020-02715-y

Landman, F. (1989). Groups: I. Linguistics and Philosophy, 12(5), 559-605

Linnebo, Ø., \& Nicolas, D. (2008). Superplurals in English. Analysis, 68(3), 186-197

\footnotetext{
$\overline{30}$ (Let $a$ be a composite object and $b$ and $c$ its proper parts. By CAI, $a a$ is identical to $b @ c$, and so $c$ included in $a a$, even though it's distinct from both $a$ and $b$.).

31 Calosi (2018: pp. 287-288) and Yi (2014: pp. 183-184; 2019: pp. 13-15) give other arguments from CAI to Nihilism, which don't obviously hinge on issues discussed in this paper. But they do rely on assumptions a defender of CAI should reject, e.g. that $x$ is included in $y$ only if $x$ is identical to $y$ (Payton 2019: pp. 18-19).
} 
Loss, R. (2020). Composition, identity, and plural ontology. Synthese. https://doi.org/10.1007/ s11229-020-02628-w

Loss, R. (2019). On atomic composition as identity. Synthese. https://doi.org/10.1007/ s11229-019-02295-6

Loss, R. (2018). A sudden collapse to nihilism. Philosophical Quarterly, 68(271), 370-375

McKay, T. J. (2006). Plural predication. Oxford University Press.

Oliver, A., \& Smiley, T. (2016). Plural logic, 2nd edition, revised and enlarged. New York: Oxford University Press.

Payton, J. D. (n.d.). Counting composites. Unpublished manuscript.

Payton, J. D. (2019). How to identify wholes with their parts. Synthese. https://link.springer.com/article/ 10.1007\%2Fs11229-019-02191-z.

Rayo, A. (2006). Beyond plurals. In A. Rayo \& G. Uzquiano (Eds.), Absolute generality. (pp. 220-254). Oxford University Press.

Schwarzchild, R. (1996). Pluralities. Kluwer Academic Publishers.

Sider, T. (2014). Consequences of collapse. In A. J. Cotnoir \& D. M. Baxter (Eds.), Composition as identity. (pp. 211-221). New York: Oxford University Press.

Sider, T. (2011). Writing the book of the world. Oxford University Press.

Sider, T. (2007). Parthood. Philosophical Review, 116(1), 51-91

Thomasson, A. L. (2014). Ontology made easy. Oxford University Press.

Uzquiano, G. (2004). Plurals and simples. The Monist, 87(3), 429-451

Wallace, M. (2011). Composition as identity, Part I. Philosophy Compass, 6(11), 804-816

Warren, J. (2016). Sider on the epistemology of structure. Philosophical Studies, 173(9), 2417-2435

Yi, B. (2019). Is composition identity? Synthese. https://doi.org/10.1007/s11229-018-02000-Z

Yi, B. (2014). Is there a plural object? In A. J. Cotnoir \& D. M. Baxter (Eds.), Composition as identity. (pp. 169-191). New York: Oxford University Press.

Yi, B. (2006). The logic and meaning of plurals. Part II. Journal of Philosophical Logic, 35, 239-288

Yi, B. (2005). The logic and meaning of plurals. Part I. Journal of Philosophical Logic, 34, 459-506

Publisher's Note Springer Nature remains neutral with regard to jurisdictional claims in published maps and institutional affiliations. 